\title{
Electrical and magnetic properties of polycrystalline Mn-doped BaTiO3 thin films grown on Pt/sapphire substrates by pulsed laser deposition
}

\author{
Yao Shuai $^{\mathrm{a}}$, Shengqiang Zhou $^{\mathrm{b}}$ and Heidemarie Schmidt ${ }^{\mathrm{C}}$
}

Forschunsgzentrum Dresden-Rossendorf e.V., Institut für lonenstrahlphysik und Materialforschung, Abteilung Halbleitermaterialien, Bautzner Landstrasse 400, 01328 Dresden, GERMANY

ay.shuai@fzd.de, bs.zhou@fzd.de, 'Heidemarie.Schmidt@fzd.de

Keywords: multiferroics, ferroelectricity, magnetism, barium titanate, bound magnetic polarons.

\begin{abstract}
Mn-doped and undoped, $200 \mathrm{~nm}$ thick $\mathrm{BaTiO}_{3}$ thin films have been grown under different oxygen partial pressures by pulsed laser deposition on Pt/sapphire substrates. X-ray diffraction (XRD) measurements reveal the same polycrystalline single-phase perovskite structure for all the thin films despite the different oxygen partial pressure, while their preferred orientation strongly depends on the oxygen partial pressure. The 5 at. \% Mn-doping decreases the dielectric loss of the Mn-doped $\mathrm{BaTiO}_{3}$ thin films, however, their relative permittivity is also decreased. Ferroelectricity has been probed on the Mn-doped and undoped $\mathrm{BaTiO}_{3}$ thin films grown under relatively high oxygen partial pressure. A ferromagnetic coupling of the $\mathrm{Mn}$ dopant ions has been probed at room tempetature on the $\mathrm{Mn}$-doped $\mathrm{BaTiO}_{3}$ thin films prepared under low oxygen partial pressure and is understood in terms of the bound magnetic polaron model.
\end{abstract}

\section{Introduction}

Much work has been carried out in the field of perovskite oxides due to their relatively simple structure and widespread applications including for example sensors, transducers, and memories. Among the perovskite oxides, $\mathrm{BaTiO}_{3}$ is one of the most intensively studied compounds since more than 60 years [1-5]. $\mathrm{BaTiO}_{3}$, as a typical $\mathrm{ABO} 3$ perovskite oxide whose structure can be described as a set of $\mathrm{BO}_{6}$ octahedrals arranged in a cubic pattern, with the $\mathrm{Ba}^{2+}$ ions located in the spaces between the octahedrals and the $\mathrm{Ti}^{{ }^{4+}}$ ions occupying the center of the octahedrals [6]. In order to improve the properties of $\mathrm{BaTiO}_{3}$ and to realize multi-functionality, various dopants have been introduced into bulk or thin film $\mathrm{BaTiO}_{3}$ to substitute the $\mathrm{Ba}^{2+}$ or $\mathrm{Ti}^{4+}$ ions. Wu et al. [7] experimentally demonstrated that a proper concentration of $\mathrm{Mn}$ dopants in $\mathrm{BaTiO}_{3}$ ceramics can significantly increase its dielectric constant and decrease the dielectric loss, which was accounted for by the reaction: $\mathrm{Mn}^{4+}+2 \mathrm{Ti}^{3+} \rightarrow \mathrm{Mn}^{2+}+2 \mathrm{Ti}^{4+}$. Cole et al. [8] fabricated a 5 mol \% Mg-doped $\mathrm{Ba}_{1-\mathrm{x}} \mathrm{Sr}_{\mathrm{x}} \mathrm{TiO}_{3}$ multilayer structure and showed that Mg-doping reduces the dielectric loss of $\mathrm{Ba}_{1-\mathrm{x}} \mathrm{Sr}_{\mathrm{x}} \mathrm{TiO}_{3}$ to 0.008 and yields a smoother surface compared to undoped $\mathrm{Ba}_{1-\mathrm{x}} \mathrm{Sr}_{\mathrm{x}} \mathrm{TiO}_{3}$. Lin et al . [9] prepared epitaxial Co-doped $\mathrm{BaTiO}_{3}$ thin films on $\mathrm{Nb}$-doped $\mathrm{SrTiO}_{3}$ substrates by pulsed laser deposition and observed a ferromagnetic (FM) behavior at room temperature. The largest saturated magnetization of $187 \mathrm{emu} / \mathrm{cm}^{3}$ was obtained for a Co-doped $\mathrm{BaTiO}_{3}$ thin film thickness of $7 \mathrm{~nm}$. However, polycrystalline, high concentration $\mathrm{Mn}$-doped $\mathrm{BaTiO}_{3}$ thin films grown on low-cost $\mathrm{Pt} / \mathrm{sapphire}$ substrates and their electrical and magnetic properties were rarely to be reported so far. Furthermore, there is no risk of ferromagnetic precipitate formation in Mn-doped $\mathrm{BaTiO}_{3}$.

\section{Experiment}

Here we report on the structural, magnetic and ferroelectric properties of undoped $\mathrm{BaTiO}_{3}(\mathrm{BTO})$ and 5 at.\% Mn-doped $\mathrm{BaTiO}_{3}$ (BTMO) thin films which have been prepared by pulsed laser deposition on $\mathrm{Pt} / \mathrm{c}$-sapphire substrates. High purity $\mathrm{BaTiO}_{3}(4 \mathrm{~N})$ powder and a mixture of high purity $\mathrm{BaTiO}_{3}(4 \mathrm{~N})$ and $\mathrm{MnO}_{2}(5 \mathrm{~N})$ powder have been pressed and finally sintered at $1300{ }^{\circ} \mathrm{C}$ for 12 hours to obtain the undoped and $\mathrm{Mn}$-doped $\mathrm{BaTiO}_{3}$ ceramic target, respectively. The $100 \mathrm{~nm}$ thick Pt bottom electrodes has been sputtered on c-sapphire substrates outside the PLD chamber. The BTMO films were grown by pulsed laser deposition using a $\mathrm{KrF}$ excimer laser $(\lambda=248 \mathrm{~nm})$ 
with a fixed repetition rate of $2 \mathrm{~Hz}$ and a deposition temperature of $650{ }^{\circ} \mathrm{C}$. To investigate the dependence of the electric and magnetic properties of the BTO and BTMO thin films on the ambient gas partial pressure, 0.01 and 0.1 mbar oxygen partial pressures were used. Ca. $100 \mathrm{~nm}$ and $200 \mathrm{~nm}$ thick films have been prepared using 1000 and 2000 laser pulses, respectively. After deposition, the films were in-situ post-annealed for 30 minutes at $650^{\circ} \mathrm{C}$ and subsequently cooled down to room-temperature with a cooling rate of $5 \mathrm{k} / \mathrm{min}$ under a constant oxygen partial pressure of 200 mbar. The crystal structure of the films has been analyzed by means of x-ray diffraction (XRD) using a D8 diffractometer with the CuK $\alpha$ radiation. The magnetic properties of the films have been probed by a superconducting quantum interference device magnetometer (Quantum Design MPMS). For electrical property measurements circular Au top electrodes with a diameter of $0.5 \mathrm{~mm}$ were deposited by magnetron sputtering on top of the as-prepared BTO and BTMO thin films using a metal shadow mask to form a parallel-plate capacitor with BTO or BTMO as the dielectric medium. The dielectric properties of the thin films have been analyzed by an impedance analyzer (Agilent 4294A) in the frequency range from $100 \mathrm{~Hz}$ to $100 \mathrm{kHz}$ under a driving voltage of $50 \mathrm{mV}$, the polarization-electric field loops of the films were observed using a Sawyer-Tower circuit [10] at $10 \mathrm{kHz}$.

\section{Results and Discussion}

Fig. 1 shows the XRD $\theta-2 \theta$ patterns of the $200 \mathrm{~nm}$ thick BTMO and BTO films grown on $\mathrm{Pt} / \mathrm{c}$-sapphire substrates. Only diffraction peaks corresponding to the perovskite structure have been observed, indicating that the prepared thin films have a single perovskite phase. The BTO thin film is preferential (111)-oriented (Fig. 1(a)), the BTMO thin film deposited at 0.1 mbar oxygen pressure reveals a random orientation (Fig. 1(b)), while the BTMO thin film deposited at 0.01 mbar oxygen pressure was highly (001)-oriented (Fig. 1(c)). Although the BTMO films contain 5 at \% Mn, only perovskite phases were detected in the BTMO thin films [Fig. 1 (b)(c)], revealing a full substitution of the $\mathrm{Ti}^{4+}$ ions (radius $0.068 \mathrm{~nm}$ ) by $\mathrm{Mn}^{4+}$ ions $(0.067 \mathrm{~nm})$, i.e. no Mn clusters have been formed.

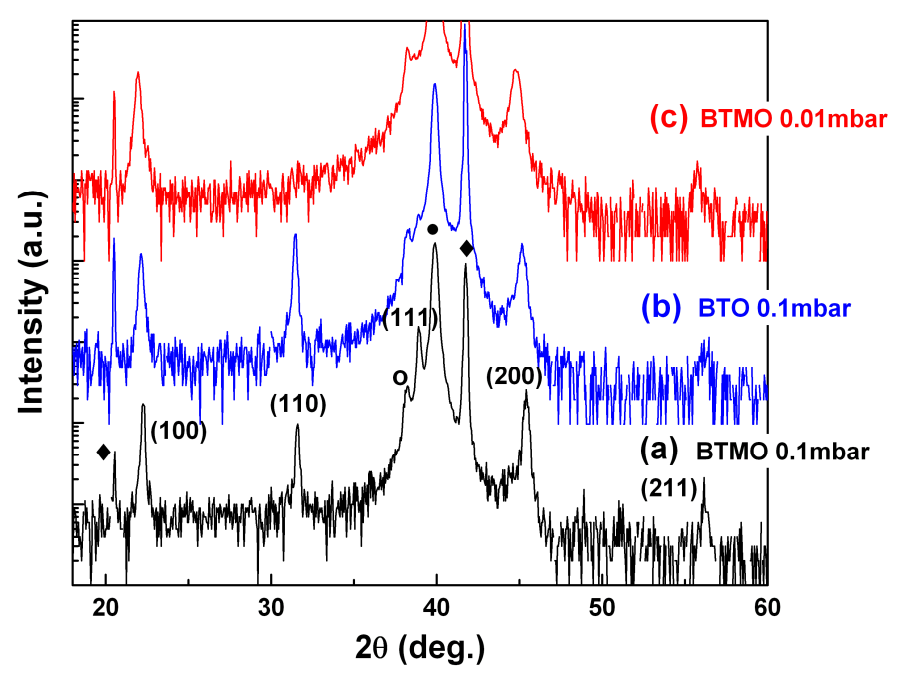

Fig. 1. XRD patterns of (a) BTMO and (b) BTO thin films prepared at 0.1 mbar oxygen pressure, and of a (c) BTMO thin film prepared at 0.01 mbar oxygen pressure on Pt/sapphire substrates. XRD peaks are labeled by the symbols $\mathrm{o}$, •, and $\bullet$ represent $\mathrm{Au}, \mathrm{Pt}$, and c-sapphire reflexes, respectively.

Fig. 2 shows the frequency dependence of the relative permittivity $\left(\varepsilon_{\mathrm{r}}\right)$ and the dielectric loss $(\tan \delta)$ at room temperature of the $200 \mathrm{~nm}$ thick BTMO and BTO thin films prepared at 0.1 mbar oxygen pressure at $650^{\circ} \mathrm{C}$. The $\tan \delta$ of the BTO film exhibits a noticeable increase with decreasing frequency from $1 \mathrm{kHz}$ to $100 \mathrm{~Hz}$. On the other hand, it can be observed that the tan $\delta$ of BTMO thin 
film showed no obvious change in the frequency range from $100 \mathrm{~Hz}$ to $100 \mathrm{kHz}$, in addition, the BTMO thin film displayed lower $\tan \delta$ than the BTO thin film in this frequency range. Chu et al. [11] have reported that Mn-doping can reduce the $\tan \delta$ of BTMO thin films, however, the maximum concentration of $\mathrm{Mn}$ in their investigation was 1 at.\%. Here we show that the tan $\delta$ of BTMO thin films can be further decreased by increasing the Mn concentration. The $\varepsilon_{\mathrm{r}}$ of the BTMO and BTO thin film is rather constant over the entire investigated frequency range, revealing a small built-in voltage at the front and bottom electrode. Therefore, the probed ferroelectricity of these thin films is an intrinsic property $[12,13]$. The $\varepsilon_{\mathrm{r}}$ of the BTMO thin films is smaller than that of the BTO thin films, indicating that excessive high concentration of Mn- doping decreases the $\varepsilon_{\mathrm{r}}$ of BTMO thin films, that is consistent with the results of BTO ceramics reported by $\mathrm{Wu}$ et al. [7]. By using a measurement frequency of $10 \mathrm{kHz}$, the $\varepsilon_{\mathrm{r}}$ and tan $\delta$ of the BTMO and BTO thin films were respectively found to be $657,1.6 \%$ and $857,2.5 \%$.

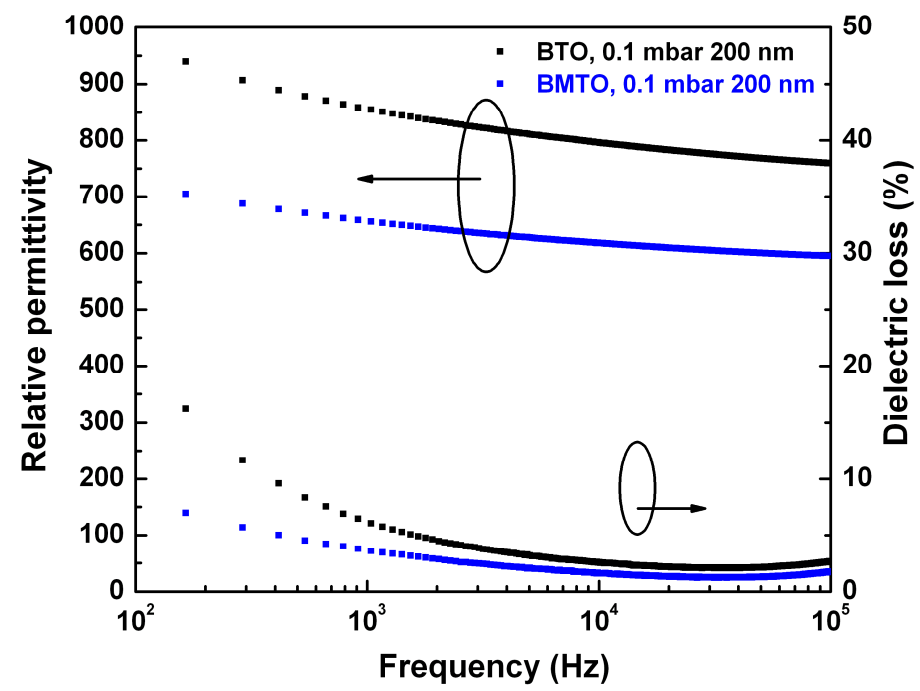

Fig. 2. Frequency dependence of the relative permittivity $\left(\varepsilon_{\mathrm{r}}\right)$ and dielectric loss $(\tan \delta)$.

The P-E loops of the BTMO and BTO thin films grown at 0.1 mbar oxygen pressure measured by sawyer-tower circuit at $10 \mathrm{kHz}$ are shown in Fig.3(a). The P-E loops indicate a typical ferroelectric (FE) behavior of both the BTMO and BTO thin films. The remnant polarization Pr and coercive electric field Ec are $2 \mathrm{Pr}=6.0 \mu \mathrm{C} / \mathrm{cm}^{2}$ and $2 \mathrm{Ec}=70 \mathrm{kV} / \mathrm{cm}$ for the $200 \mathrm{~nm}$ thick BTMO film and $2 \mathrm{Pr}=9.6 \mu \mathrm{C} / \mathrm{cm}^{2}$ and $2 \mathrm{Ec}=86 \mathrm{kV} / \mathrm{cm}$ for the $200 \mathrm{~nm}$ thick BTO film. The $2 \mathrm{Pr}$ value of the BTMO thin film is larger than that of other reported pure polycrystalline BTO thin films grown on different substrates [14-16], indicating a good crystallization quality of the obtained BTMO thin film. For the $100 \mathrm{~nm}$ thick BTMO thin film an even larger remnant polarization of $2 \mathrm{Pr}=10.2$ $\mu \mathrm{C} / \mathrm{cm}^{2}$ has been observed. This value is comparable to the that of the reported epitaxial (001)-oriented 1 at.\% Mn-doped BTO thin film [17], while the coercive electric field was also increased (Fig.3(b)). The increased 2Pr of the $100 \mathrm{~nm}$ thick BTMO thin film is due to the larger electric field applied on the thin films with a smaller thickness, such that more ferroelectric domains can be reversed under higher electric field. It should be noticed that even under a high electric field of $800 \mathrm{kV} / \mathrm{cm}$, the BTMO thin films were not broken down and a saturated P-E loop can still be observed, probably arising from the presence of few defects in the $100 \mathrm{~nm}$ thick BTMO films. However, for the $200 \mathrm{~nm}$ thick BTMO film grown at 0.01 mbar oxygen pressure no saturated P-E loop can be observed (not shown), which is probably due to the large leakage current resulting from oxygen vacancies. 

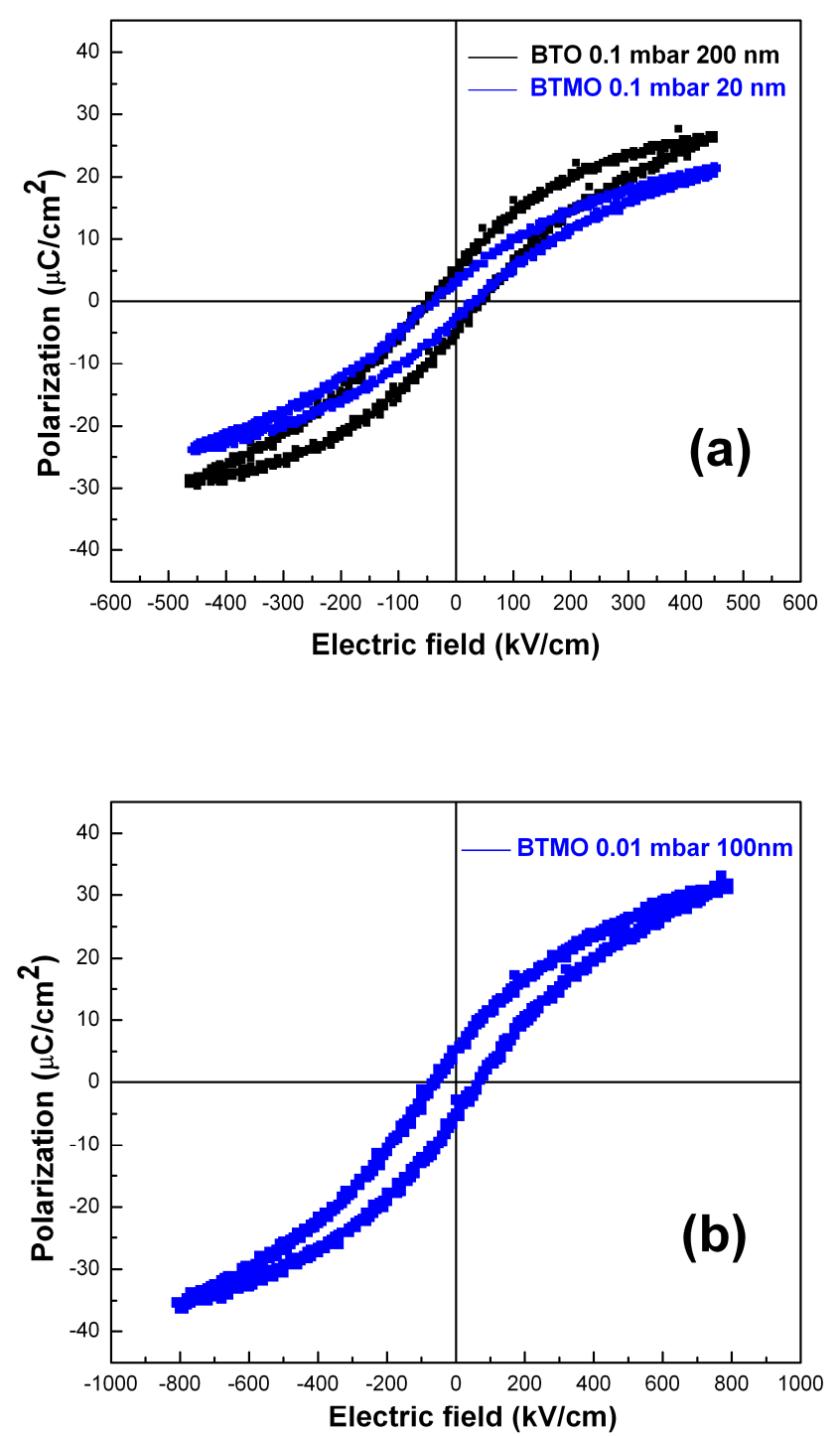

Fig. 3. Polarization-electric field hysteresis loops of (a) the BTMO (200nm) and BTO (200 nm) thin films and of the (b) BTMO $(100 \mathrm{~nm})$ thin film grown at 0.1 mbar oxygen pressure.

The magnetization-field (M-H) hysteresis loop is shown in Fig.4 for the BTMO thin film deposited at 0.01 mbar oxygen pressure and reveals a ferromagnetic (FM) coupling of the Mn dopant ions, whereas the BTMO thin film grown at $0.1 \mathrm{mbar}$ oxygen pressure is not ferromagnetic. As seen from the XRD pattern in Figure 1(c), no XRD peak corresponding to a non-perovskite structure can be observed, excluding the Mn segregation as the origin of the observed FM behavior of the BTMO thin film grown in low oxygen pressure. One of the possible reasons of the FM behavior in BTMO thin films can be explained by a bound magnetic polaron model (BMP) which has been proposed by Coey et al. [18]. In this model, a coupling effect between electrons bound to oxygen vacancies and $\mathrm{Mn}^{4+}$ dopant ions is regarded as the origin of ferromagnetism in Mn-doped BTO thin films. By the BMP model, we can also understand the reason why only the BTMO thin film grown in low oxygen pressure (0.01 mbar) shows FM behavior, however, the BTMO thin film prepared in high oxygen pressure $(0.1 \mathrm{mbar})$ is non-ferromagnetic, that is because the former has more oxygen vacancies inside due to the low deposition pressure, giving rise to an enhancement of the exchange interaction between the $d$ spins in the $\mathrm{Mn}^{4+}$ and the electrons bound to oxygen vacancies. This can also be proved by P-E loop measurements. The BTMO film grown at low oxygen pressure reveals a large leakage current so that no typical P-E curve can be observed. This is ascribed to the large amount of 
oxygen vacancies, while P-E loops can be probed on the BTMO thin film prepared at high oxygen pressure as shown in Fig. 3. Note that for the BTMO thin film grown at low oxygen pressure, the magnetic moment is a superposition of the paramagnetic magnetization from isolated $\mathrm{Mn}^{4+}$ outside the BMPs and ferromagnetic magnetization from $\mathrm{Mn}^{4+}$ inside the BMPs. The paramagnetic component disappears at $150 \mathrm{k}$, while the ferromagnetism component is nearly temperature independent.
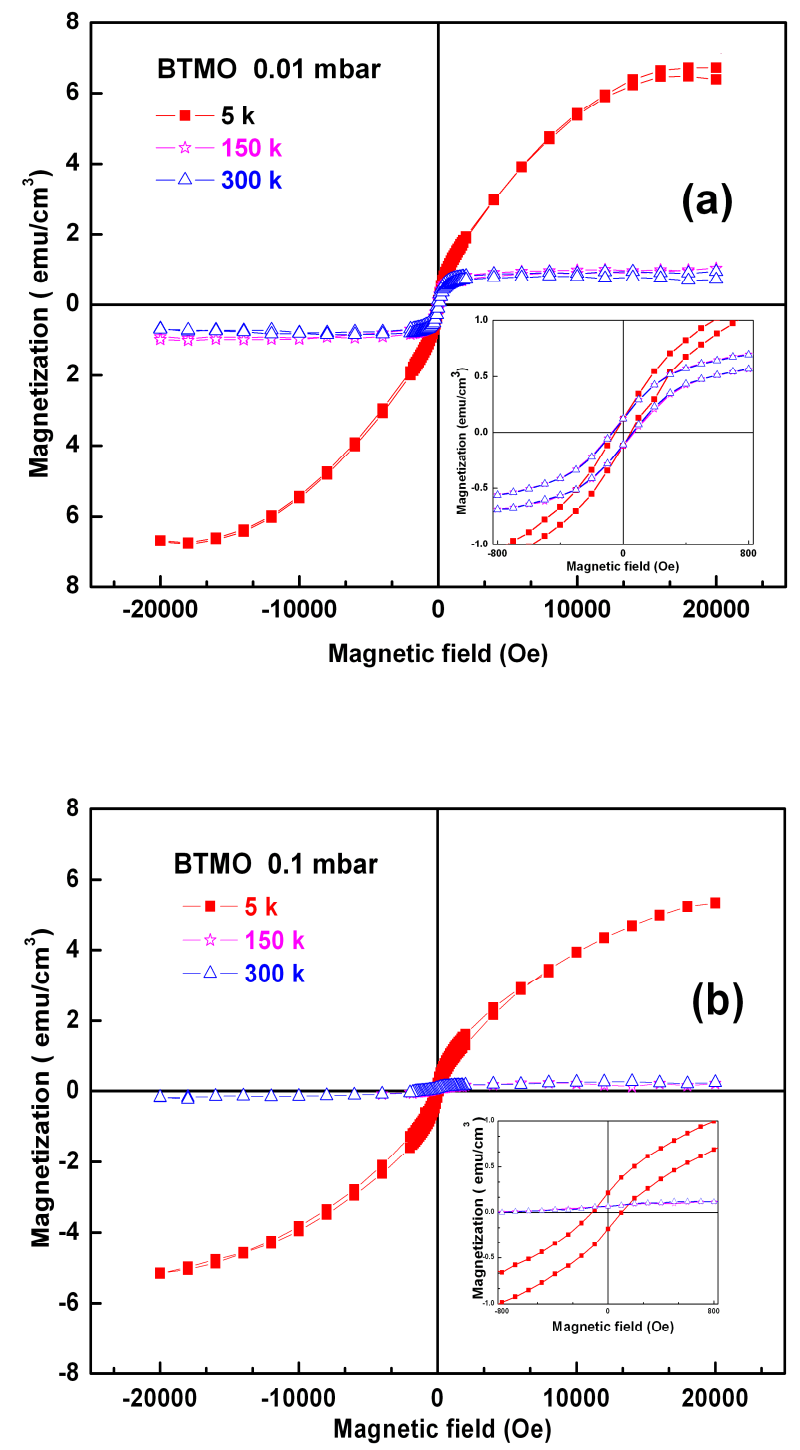

Fig. 4. Magnetization-field hysteresis loop of the BTMO thin films at different oxygen pressure. The inset shows a zoom in low magnetic fields manifesting a hysteresis of the loops. For the BTMO 0.1 mbar, only half loop was measured at 150 and $300 \mathrm{k}$ (Fig. 4(b)).

\section{Summary}

In conclusion, 5 at.\% Mn-doped $\mathrm{BaTiO}_{3}$ and pure $\mathrm{BaTiO}_{3}$ thin films were prepared on $\mathrm{Pt} /$ sapphire substrates at different oxygen pressures by pulsed laser deposition. The films grown at $0.1 \mathrm{mbar}$ oxygen pressure show saturated P-E curves, indicating a typical ferroelectricity. However, the Mn-doped $\mathrm{BaTiO}_{3}$ films grown at 0.01 mbar oxygen pressure reveal no P-E curve probably due to oxygen vacancies causing large leakage currents. The Mn-doped $\mathrm{BaTiO}_{3}$ thin film prepared at low oxygen pressure reveals ferromagnetic coupling at room temperature, which can be explained by a bound magnetic polaron model. Those bound electrons are necessary for mediating the parallel alignment of $\mathrm{Mn}^{4+}$ dopant ions but decrease in the same time the breaktrough voltage of the BTMO 
capacitors. The result indicates the possibility to introduce ferromagnetism into ferroelectric polycrystalline BTMO thin films on low-cost Pt/sapphire substrates, however, further investigations are necessary to realize the coexistence of ferromagnetism and ferroelectricity in polycrystalline BTMO thin films.

Acknowledgements

One of the authors (Yao Shuai) would like to thank the China Scholarship Council (grant number: 2009607011).

\section{References}

[1] M.M. Vijatovic, J.D. Bobic and B.D. Stojanovic: Sci. Sintering Vol. 40 (2008), p. 155.

[2] H.F Cheng et al: Jpn. J. Appl. Phys Vol. 32 (1993), p. 5656.

[3] J.D. Baniecki et al: J. Appl. Phys Vol. 97 (2005), p. 114101.

[4] N. Yanase et al: Jpn. J. Appl. Phys Vol. 38 (1999), p. 5305.

[5] K.J. Choi et al: Science Vol. 306 (2004), p. 1005.

[6] N. Izyumskaya et al: Crit. Rev. Solid State Mater. Sci Vol. 32 (2007), p. 111.

[7] S. Wu et al: J. Mater. Sci: Mater Electron Vol. 19 (2008), p. 505.

[8] M.W. Cole et al: Appl. Phys. Lett Vol. 92 (2008), p. 072906

[9] Y.H. Lin et al: Appl. Phys. Lett Vol. 92 (2008), p. 112501.

[10] C.B. Sawyer and C.H. Tower: Phys. Rev Vol. 35 (1930), p. 269.

[11] J.P. Chu et al: J. Mater Sci: Vol. 42 (2007), p. 346.

[12] J. Wu et al: Appl. Phys. Lett Vol. 94 (2009), p. 172906.

[13] D. Shindo, T. Yoshimura and N. Fujimura: Appl. Surf. Sci Vol. 254 (2008), p. 6218.

[14] R. Thomas et al: J. Appl. Phys Vol. 90 (2001), p. 1480.

[15] L. Qiao and X.F. Bi: J. Eur. Ceram. Soc Vol. 29 (2009), p. 1995.

[16] L.M. Huang et al: J. Appl. Phys Vol. 100 (2006), p. 034316.

[17] Y.H. Lin et al: Appl. Phys. Lett Vol. 95 (2009), p. 033105.

[18] J.M.D. Coey, M. Venkatesan, C.B. Fitzgerald: Nat. Mater Vol. 4 (2005), p. 173. 